\title{
Erectile dysfunction and testosterone levels prior to COVID-19 disease: What is the relationship?
}

\author{
Kadir Karkin, Ergün Alma \\ Department of Urology, Health Sciences University, Adana City Training and Research Hospital, Adana, Turkey.
}

\begin{abstract}
Summary Objective: We aimed to investigate the relationship between COVID-19 and Erectile

Dysfunction (ED) and the effect of serum testosterone level on the disease prognosis.

Methods: Between April-December 2020, 70 patients who were admitted with a complaint of ED after having COVID-19 and whose serum testosterone level was checked for varicocele, premature ejaculation, and infertility reasons before COVID-19. The patients filled the International Index of Erectile Function (IIEF-5) and their testosterone level was checked. The questionnaire was arranged to assess the first month before COVID-19 and after COVID-19. Testosterone levels of the patients before and after COVID-19 were compared. The relationship between testosterone levels and hospitalization in the intensive care was evaluated.

Results: It was revealed that testosterone levels and IIEF-5 scores after COVID-19 in all patients were statisticaly and significantly different compared to the period before COVID-19 $(p<0.05)$. Testosterone levels of patients in need of intensive care were significantly higher than those without any need of intensive care $(p<0.05)$.

Conclusions: Our study has presented that COVID-19 may cause ED and high testosterone levels increase the rate of hospitalization in the intensive care by intensifying the disease.
\end{abstract}

KEY WORDS: COVID-19; Erectile dysfunction; Testosterone.

Submitted 18 September 2021; Accepted 9 October 2021

\section{INTRODUCTION}

The global coronavirus disease (COVID-19) results in a cytokine storm that leads to the development of microthrombosis and diffuse intravascular coagulation (DIC) (1). Despite the fact that lungs are the organs which are primarily targeted in cytokine storm, the cardiovascular system is also affected. The autopsies which were performed showed that there were signs of endothelial damage, pulmonary embolism, bleeding at multiple levels including alveolar, microangiopathy, and vascular dysfunctions in the form of vasculitis (2).

The relationship between Erectile Dysfunction (ED) and COVID-19 develops due to vasculogenic and hormonal causes which were caused by the primary disease. The vasculogenic damage and ED are also seen as predictors of a cardiovascular pathology which may be observed in patients in the future (3). Testosterone, which is the most important hormone in male erection, modulates almost every component involved in erectile function, from the pelvic ganglia to smooth muscles and the endothelial cells of the corpora cavernosa (4). Low testosterone levels are associated with decreased libido, ED, decreased energy, depressive symptoms, and fatigue (5). Changes in testosterone levels after COVID-19 may not only increase the severity of ED but also cause ED alone (6).

The relationship between COVID-19 and testosterone is based on contracting the disease, the severity of the disease and progression of the disease. COVID-19 makes use of Transmembrane protease serine 2 (TMPRSS2) to penetrate into the host cell and androgen receptors are transcription supportive for TMPRSS2. The higher the testosterone level, the easier it is for COVID-19 to penetrate into the host via this mechanism (7). Besides, TMPRSS2 is up-regulated in prostate cancer where it supports tumor progression, thus these patients may have a higher risk of SARS-COV-2 infection. TMPRSS2 inhibitors may be useful for the treatment or prevention of COVID-19 (8). On the contrary, some other studies have reported that low testosterone levels are associated with acute respiratory distress syndrome (ARDS) by increasing proinflammatory cytokines and they intensify COVID-19 infection in elderly men (9).

ED which is caused by COVID-19 appears to be associated with both vascular endothelial damage and reduction in testosterone level. Even though the studies in the last year mostly focused on primary disease due to the pandemic, In some studies, COVID-19 and its effects on sexual life have begun to be revealed and suggestions are made in terms of sexual health, especially through andrology associations (10), but the results on effects of COVID-19 infection on the male reproductive system are currently insufficient as they are based on a small number of patients and therefore are often contradictory (11).

Our study aimed to figure out the factors which may play a role in the development of ED in our patients with COVID-19 and the effect of testosterone level on the prognosis of COVID-19.

\section{MATERIALS AND METHOdS}

Study design and patients

Our study has been designed after being approved by the Republic of Turkey Ministry of Health and the local ethics committee (Ethical committe approval number: $1303 / 27.01 .2021$ ) and 210 patients who were admitted 
to our clinic with a complaint of ED between AprilDecember 2020 were evaluated prospectively. Written consent forms were obtained from all patients who participated in the study. In order to figure out the erectile conditions of the patients, the IIEF-5 questionnaire, fiveitem short version of the international index of erectile function (IIEF-EF), was used. This questionnaire was designed in a form assessing the first month before COVID-19 infection and the period after COVID-19. According to this form, scores between 5-7 were considered as severe, 8-17 as moderate, 17-21 as mild and 22-25 as no ED.

Male patients with an elapsed time at least six months after COVID-19 and who did not have ED according to the IIEF-5 score before COVID-19 infection, and whose testosterone level was measured for varicocele, premature ejaculation, infertility reasons in the last year were included in our study. Furthermore, Beck depression scale was used to determine the current depressive states of the patients. Patients with minimal depression (0-9) according to this scale were included in the study. The patients who had comorbidities before COVID-19 such as obesity, hypertension, diabetes or heart disease, the patients who have used drugs (such as beta blocker) that can cause ED during and after COVID-19, the patients with who had an increase in smoking and alcohol use after COVID-19, the patients with an elapsed time of less than 6 months after COVID-19, the patients whose testosterone levels were not known before COVID-19, the patients who did not have a minimal depression level according to the Beck depression scale, the patients with an IIEF-5 score of 21 and below, the patients who had treatment for ED before COVID-19, whose detailed medical history could not be taken and who did not have education level enough to fill in IIEF-5 form were not included in the study. After being evaluated according to the exclusion criteria, our study was carried on with 70 patients.

The ages of the patients, their results of COVID-19 polymerase chain reaction (PCR) test, testosterone level, reports consistent with COVID-19 observed in thoracic computed tomography (CT) and the presence of hospitalization in intensive care unit were recorded. The patients were evaluated after being classified under three different groups according to their ages. The patients aged between 20-40 were recorded in group 1 , the ones aged between 40-60 were recorded in group 2 and the ones aged 60 or over were recorded in group 3 . The levels of serum testosterone before and after COVID-19 were compared among the groups. The relationship between the levels of testosterone and the hospitalization in the intensive care unit and ED states of the patients hospitalized in the intensive care unit were evaluated.

\section{Data collection}

The electronic hospital information system was used to get epidemiological data, including demographic, clinical and laboratory findings. Considering the circadian rhythm of testosterone release, the total testosterone which were measured in the venous blood sample between 7 and 11 in the morning were recorded. Peripheral venous blood samples were evaluated in the Central Laboratory of Adana City Training and Research Hospital by using the standard pro- cedures. Biochemical hormonal parameters were measured by Siemens ADVIA 1800 automatic biochemistry analyser (Siemens Healthcare Diagnostics Inc, Laboratory Diagnostics, Advia Centaur XPT, Erlangen, Germany, manufactured in Ireland).

\section{Statistical analysis}

SPSS 23.0 package program (IBM, Armonk, NY) was used in the statistical analysis of data. Categorical measurements were summarized as numbers and percentages, continuous measurements were summarized as mean, standard deviation and minimum-maximum. The appropriateness of the variables to normal distribution was investigated by using Kolmogorov-Smirnov/Shapiro-Wilk Tests. Mann-Whitney U test and Wilcoxon test were used in the groups which do not agree with normal distribution. Spearman correlation analysis was benefited from in the comparison of the relationship between the numerical variables. Statistical significance level was taken as 0.05 in all tests.

\section{RESULTS}

The mean age in our study was $52.3 \pm 13.5$ years. When the groups were considered, it was seen that $22.9 \%(\mathrm{n}=$ 16) of the patients were in group $1,47.1 \%(n=33)$ were in group 2 and $30 \%(n=21)$ were in group 3 . The mean IIEF score of the patients was 23 before COVID-19 and 11.03 after COVID-19 (Table 1).

No statistical difference was observed between the patient's age and the rate of hospitalization in the intensive care unit $(p>0.05)$. A statistically significant difference was observed between the IIEF scores of the patients with and without intensive care need in the period after COVID-19 $(\mathrm{p}<0.05)$.

Testosterone levels of patients with intensive care need were significantly higher than those of the patients without intensive care need $(\mathrm{p}<0.05)$ (Table 2).

Testosterone levels after COVID-19 were found significantly lower than the testosterone levels before COVID19 ( $\mathrm{c}<0.001)$. In all three groups, it was seen that testosterone levels and IIEF scores after COVID-19 were statistically and significantly different compared to the ones before COVID-19 $(\mathrm{p}<0.05)$. As the age of the patients increased, IIEF scores after COVID-19 and testosterone levels before and after COVID-19 were found to be significantly lower $(\mathrm{p}<0.05)$. Also, there was a statistically significant relationship between IIEF scores and testosterone levels $(\mathrm{p}<0.05)$. While there was a positive corre-

Table 1.

Demographic data and mean IIEF according to the patient groups.

\begin{tabular}{|l|c|c|}
\hline Age & Frequency $(\mathbf{n})$ & Percentage (\%) \\
\hline $20-40$ & 16 & 22.9 \\
$40-60$ & 33 & 47.1 \\
60 and over & 21 & 30 \\
\hline & Mean \pm SD & Mediann (min-max) \\
\hline Age & $52.33 \pm 13.51$ & $56(22-74)$ \\
IIEF Score before COVID & $23.5 \pm 1.57$ & $23(22-25)$ \\
IIEF Score after COVID & $11.03 \pm 3.76$ & $11(5-18)$ \\
\hline
\end{tabular}


Table 2.

Rates of hospitalization in the intensive care according to groups, evaluation of hospitalization in the intensive care and IIEF score before and after COVID-19 and correlation between the hospitalization in the intensive care and testosterone level.

\begin{tabular}{|c|c|c|c|c|c|}
\hline & & $\begin{array}{c}20-40 \\
(n=16) \\
n(\%)\end{array}$ & $\begin{array}{c}40-60 \\
(n=33) \\
n(\%)\end{array}$ & $\begin{array}{c}60 \text { and over } \\
(n=21) \\
n(\%)\end{array}$ & $\mathbf{P}$ \\
\hline Hospitalization in the intensive care & $\begin{array}{l}\text { Yes } \\
\text { No }\end{array}$ & $\begin{array}{r}5(31.2) \\
11(68.8)\end{array}$ & $\begin{array}{l}11(33.3) \\
22(66.7)\end{array}$ & $\begin{array}{l}6(28.6) \\
15(71.4)\end{array}$ & .93 \\
\hline Hospitalization in the intensive care & $\begin{array}{l}\text { No } \\
\text { Yes } \\
p\end{array}$ & $\begin{array}{c}\text { IIEF score before } \\
\text { Median (min- } \\
24(22-25) \\
23(22-24) \\
.95\end{array}$ & $\begin{array}{ll}\text { OVID } & \text { IIE } \\
\text { ax) } & M\end{array}$ & $\begin{array}{c}\text { F score after COVID } \\
\text { edian (min-max) } \\
13(5-18) \\
8(5-13)\end{array}$ & $\begin{array}{c}\mathbf{P} \\
<.001 \\
<.001 \\
<.001\end{array}$ \\
\hline Hospitalization in the Intensive care & $\begin{array}{l}\text { No } \\
\text { Yes }\end{array}$ & \multicolumn{2}{|c|}{$\begin{array}{l}\text { Testosterone level } \\
\text { Median (min-max) }\end{array}$} & & $\begin{array}{c}\mathbf{P} \\
<.001\end{array}$ \\
\hline
\end{tabular}

Table 3.

Comparison of the IIEF scores and testosterone levels before and after COVID-19, evaluation of the IIEF scores and testosterone levels before and after COVID-19 according to groups and correlation between testosterone level, age and IIEF score.

\begin{tabular}{|c|c|c|c|c|}
\hline & \multicolumn{3}{|c|}{ Mean \pm SD } & P \\
\hline IIEF Score before COVID & \multicolumn{3}{|c|}{$23.5 \pm 1.57$} & $<.001$ \\
\hline IIEF Score after COVID & \multicolumn{3}{|c|}{$11.03 \pm 3.76$} & \\
\hline Testosterone level before COVID & \multicolumn{3}{|c|}{$345.67 \pm 109.48$} & $<.001$ \\
\hline Testosterone level after COVID & \multicolumn{3}{|c|}{$297.31 \pm 96.04$} & \\
\hline According to groups & $\begin{array}{c}20-40 \\
(n=16) \\
\text { Median (min-max) }\end{array}$ & $\begin{array}{c}40-60 \\
(n=33) \\
\text { Median (min-max) }\end{array}$ & $\begin{array}{c}60 \text { and over } \\
\text { ( } n=21) \\
\text { Median (min-max) }\end{array}$ & $\mathbf{P}$ \\
\hline IIEF score before COVID & $24(23-25)$ & $23(22-25)$ & $22(22-23)$ & .9 \\
\hline IIEF score after COVID & $14(6-18)$ & $11(5-18)$ & $8(5-17)$ & $<.001$ \\
\hline Testosterone before COVID & 400 (213-531) & $390(122-680)$ & $225(112-423)$ & $<.001$ \\
\hline \multirow[t]{3}{*}{ Testosterone after COVID } & $300(230-449)$ & $300(100-600)$ & $200(100-400)$ & $<.001$ \\
\hline & \multicolumn{2}{|c|}{ Testosterone level } & \multicolumn{2}{|c|}{ Age } \\
\hline & $r$ & $p$ & $r$ & $p$ \\
\hline IIEF score before COVID & 0.383 & .001 & -0.675 & $<.001$ \\
\hline IIEF score after COVID & 0.423 & $<.001$ & -0.557 & $<.001$ \\
\hline
\end{tabular}

lation between the testosterone level findings, the IIEF scores before COVID-19 $(\mathrm{r}=0.383)$ and the IIEF scores after COVID-19 $(r=0.423)$ of the patients who participated in the study, there was a negative correlation between the ages of the patients and the IIEF scores before COVID-19 $(\mathrm{r}=-0.675)$ and the IIEF scores after COVID-19 $(\mathrm{r}=-0.557)(\mathrm{p}<0.05)($ Table 3$)$.

\section{Discussion}

We showed with this study that COVID-19 causes ED in all age groups, reducing testosterone levels seriously. Moreover, we also oberved that the higher the testosterone levels during COVID-19, the more severe the disease progresses. To the best of our knowledge, a similar study suggesting that COVID-19 causes ED has not been published before in the related literature.
ED is multidimensional and commonly male sexual dysfunction involves a change in any component of the erectile response, including organic, relational, and psychological. Non-endocrine (neurogenic, vasculogenic, and iatrogenic) and endocrine pathways may cause ED (12, 13). Endothelial dysfunction equals erectile dysfunction or vice versa (ED-ED). It is presented in the literature that the prevalence of ED can be up to 30\% in young men and that the rare of ED is further increased in elderly due to diseases of coronary and penile arteries (14-16).

In COVID-19 infection, the ACE enzyme, which provides access to host cells, is expressed by endothelial cells and the fragile vascular bed is affected preparing the ground for the development of ED (17-22).

Testosterone deficiency is one of the most important hormonal causes of ED development. Testosterone modulates almost all components of the erectile mechanism, down to the endothelial cells in the corpus cavernosum. Current EAU guidelines recommend to measure baseline testosterone levels in all men who admit with a complaint of ED (7). Araujo et al. showed in their studies that the testosterone levels of 24\% of men aged between 30-79 were below 300 and $5.6 \%$ of them had symptomatic androgen deficiency (23). Yassin et al. reported that testosterone replacement therapy (TRT) improved erectile function in hypogonadal men who previously received phosphodisterase-5 inhibitor (PDE5I) before (24). Rizk et al. suggested that the use of TRT as monotherapy in patients with mild ED was effective (25). Our present study shows that patients of all age groups are affected, although ED which develops after COVID-19 seems to affect more elderly patients. It was also revealed that testosterone levels of the patients were significantly lower in all age groups, especially in elderly patients.

In COVID-19 infection, Leydig cells in the testicles are damaged and this testicular damage causes a decrease in serum testosterone levels (26). Ling Ma et al. reported that testosterone/luteinizing hormone (LH) ratios were significantly lower in patients with COVID-19 infection (27). Vanhorebeek et al. observed that there was a decrease in testosterone level in the acute phase of COVID-19 (28). Also Okçelik reported that testosterone levels decreased during acute COVID-19 infection and especially in patients with pulmonary involvement (29). It was also concluded in our study that the significant decrease in the testosterone levels of patients after COVID-19 is an indicator of a possible testicular damage and this finding supports the studies in the literature.

Testosterone is a hormone which has an effect not only on the erection mechanism but also on many functions in the body. Inflammation is known to be suppressed as a result of the decrease in pro-inflammatory cytokines and the increase of anti-inflammatory cytokines while responding to infections. It was shown in the study of Mohamad et al. that testosterone suppresses inflammation by increasing anti-inflammatory cytokines and decreasing pro-inflammatory cytokines (30). Malkin et al. conducted a crossover study with 27 men with symptomatic androgen deficiency. They concluded that testosterone treatment decreased pro-inflammatory cytokine levels and increased the concentration of interleukins, which are some of the anti-inflammatory cytokines (31). Although 
our study did not focus on any evaluation in this direction, we assume that the low testosterone level in patients after COVID-19 may have contributed to inflammation. We also believe that the investigation of the effect of TRT on the clinical findings of the patients should be supported by prospective studies.

During the cytokine storm caused by COVID-19, low testosterone can also affect the prognosis of the disease. Jiawei et al. have suggested in their study that testosterone may play a role in the chain of events leading to the progression of COVID-19 infection due to the cytokine storm. It was concluded with this study that there may be a negative correlation between ACE2 expression and COVID-19 mortality as a result of suppression of ACE2 expression by inflammatory cytokines accompanying the decrease in androgens and oestrogens in the elderly (32). Giagulli et al. has shown that testosterone can make men susceptible to a common COVID-19 infection compared to oestrogen, and low serum testosterone levels in severe patients make men, especially older, susceptible to poor prognosis or death (33). Papadopoulos et al. reported that low testosterone levels are associated with ARDS increasing the severity of COVID-19 infection in elderly men and they added that normal testosterone levels may also provide some slight protection against COVID-19 (13). Similar studies in the literature support a relationship between the need for intensive care and low testosterone levels in COVID-19 infection $(34,35)$. Studies have also been reported that testosterone affects by using a different mechanism apart from the cytokine mechanism during COVID-19 infection. It has been reported in the study of Lii et al. that the first biological step which is necessary for the potential infectivity of COVID-19 is to activate ACE2 as the entry receptor and to use cellular transmembrane protease serine 2 (TMPRSS2) to prepare spike protein (36). Hoffmann et al. showed in their study that viral spread and pathogenesis are provided in infected hosts by spike proteins becoming ready by TMPRSS2 (37). Heurich et al. presented in their study that TMPRSS2 can also break down ACE2 for viral entry and that androgen receptor activity is a requirement for the transcription of the TMPRSS2 gene (38). There are studies reporting that high testosterone levels are associated with poor prognosis in COVID-19 through this mechanism. Wambier et al. reported that the hyperandrogenic phenotype may be associated with increased viral load, increased viral spread, and severity of lung involvement for COVID-19 (39). It was reported by Rozhivanov et al. that both TMPRSS2 expression and a more severe course of coronavirus infection were observed in men with hyperandrogenism. In this context, some researchers suggested the creation of androgen deprivation with drug treatments for men at high risk of developing COVID-19 (40). There are some contrasting views in the literature on the effect of testosterone level on COVID-19 prognosis and further studies are required to support them. The results of our study showed that patients with high testosterone levels need intensive care more and this supports the studies in the literature claiming that high testosterone level is a poor prognostic factor for COVID-19.

There are some limitations of our study. Our study reflects the results of one centre and includes a limited number of patients. Besides, although we have excluded many factors that cause ED (such as obesity, hypertension, diabetes or heart disease), the ED mechanism is dependent on a lot of factors that our study may not have been able to evaluate adequately since a non-ED vital disease is discussed. Other limitations are that the psychological state of the patients may have affected the IIEF-5 score, and conditions such as varicocele and infertility may cause low testosterone levels, which may affect our results.

However, to the best of our knowledge, our study is one of the first studies showing that COVID-19 causes erectile dysfunction and there are only a limited number of studies in the literature on this subject. We hope that our study will make a considerable contribution to the literature with this aspect.

\section{Conclusions}

COVID-19 can cause erectile dysfunction in men of all age groups, and high testosterone increases the rate of hospitalization in the intensive care unit by intensifying the disease.

\section{ACKNOWLEDGMENT}

We would like to thank the Adana City Hospital Urology Clinic for their contribution.

\section{RefEREnCES}

1. Jose RJ, Manuel A. COVID-19 cytokine storm: the interplay between inflammation and coagulation. Lancet Respir Med 2020; 8:e46-e47.

2. Menter T, Haslbauer JD, Nienhold R, et al. Postmortem examination of COVID-19 patients reveals diffuse alveolar damage with severe capillary congestion and variegated findings in lungs and other organs suggesting vascular dysfunction. Histopathology. 2020; 77:198-209.

3. Jannini EA. SM = SM: The interface of systems medicine and sexual medicine for facing non-communicable diseases in a genderdependent manner. Sex Med Rev. 2017; 5:349-364.

4. Isidori AM, Buvat J, Corona G, et al. A critical analysis of the role of testosterone in erectile function: from pathophysiology to treatment-a systematic review. Eur Urol. 2014; 65:99-112.

5. Schubert M, Jockenhovel F. Late-onset hypogonadism in the aging male (LOH): definition, diagnostic and clinical aspects. J Endocrinol Invest. 2005; 28:23-27.

6. Blute M, Hakimian P, Kashanian J, et al. Erectile dysfunction and testosterone deficiency. Front Horm Res. 2009; 37:108-122.

7. Mohamed MS, Moulin TC, Schiöth HB. Sex differences in COVID19: the role of androgens in disease severity and progression. Endocrine. 2021; 71:3-8.

8. Rodriguez Bustos H, Bravo Maturana G, Cortés-Chau F, et al. Effects of COVID-19 on male sex function and its potential sexual transmission. Arch Ital Urol Androl. 2021; 93:48-52.

9. Papadopoulos V, Li L, Samplaski M. Why does COVID-19 kill more elderly men than women? Is there a role for testosterone? Andrology. 2021; 9:65-72. 
10. Maretti C, Privitera S, Arcaniolo D, et al. COVID-19 pandemic and its implications on sexual life: Recommendations from the Italian Society of Andrology. Arch Ital Urol Androl. 2020; 92:73-77

11. Delle Fave RF, Polisini G, Giglioni G, et al. COVID-19 and male fertility: Taking stock of one year after the outbreak began. Arch Ital Urol Androl. 2021; 93:115-119.

12. Yafi FA, Jenkins L, Albersen M, et al. Erectile dysfunction. Nat Rev Dis Primers. 2016; 2:16003.

13. Irwin GM. Erectile dysfunction. Prim Care. 2019; 46:249-255.

14. Guay AT. ED2: erectile dysfunction = endothelial dysfunction. Endocrinol Metab Clin North Am. 2007; 36:453-463.

15. Mola JR. Erectile dysfunction in the older adult male. Urol Nurs. $2015 ; 35: 87-93$

16. Nguyen HMT, Gabrielson AT, Hellstrom WJG. Erectile dysfunction in young men-A review of the prevalence and risk factors. Sex Med Rev. 2017; 5:508-520.

17. Varga Z, Flammer AJ, Steiger P, et al. Endothelial cell infection and endotheliitis in COVID-19. Lancet 2020; 395:1417-1418.

18. Maiorino MI, Bellastella G, Giugliano D, et al. From inflammation to sexual dysfunctions: a journey through diabetes, obesity, and metabolic syndrome. J Endocrinol Invest. 2018; 41:1249-1258.

19. Zhang $\mathrm{H}$, Penninger JM, Li Y, et al. Angiotensin-converting enzyme 2 (ACE2) as a SARS-CoV-2 receptor: molecular mechanisms and potential therapeutic target. Intensive Care Med. 2020; 46:586590.

20. Zhou P, Yang XL, Wang XG, et al. A pneumonia outbreak associated with a new coronavirus of probable bat origin. Nature. 2020; 579:270-273.

21. Hamming I, Timens W, Bulthuis ML, et al. Tissue distribution of ACE2 protein, the functional receptor for SARS coronavirus. A first step in understanding SARS pathogenesis. J. Pathol. 2004; 203:631-637.

22. Lovren F, Pan Y, Quan A, et al. Angiotensin converting enzyme2 confers endothelial protection and attenuates atherosclerosis. Am. J. Physiol. Circ. Physiol. 2008; 295: H1377-H1384.

23. Araujo AB, Esche GR, Kupelian V, et al. Prevalence of symptomatic androgen deficiency in men. J Clin Endocrinol Metab. 2007; 92:4241-7.

24. Aksam A Yassin, Farid Saad. Testosterone and erectile dysfunction. J Androl. 2008; 29:593-604.

25. Rizk PJ, Kohn TP, Pastuszak AW, Khera M. Testosterone therapy improves erectile function and libido in hypogonadal men. Curr Opin Urol. 2017; 27:511-515.

26. Douglas GC, O'Bryan MK, Hedger MP, et al. The novel angiotensin-converting enzyme (ACE) homolog, ACE2, is selectively expressed by adult Leydig cells of the testis. Endocrinology. 2004; 145:4703-4711.

27. Ma L, Xie W, Li D, et al. Effect of SARS-CoV-2 infection upon male gonadal function: A single center-based study. MedRxiv; 2020.

28. Vanhorebeek I, Langouche L, Van den Berghe G. Endocrine aspects of acute and prolonged critical illness. Nat Clin Pract Endocrinol Metab. 2006; 2:20-31.

29. Okçelik S. COVID-19 pneumonia causes lower testosterone levels. Andrologia. 2021; 53:e13909.

30. Mohamad NV, Wong SK, Wan Hasan WN. The relationship between circulating testosterone and inflammatory cytokines in men. Aging Male. 2019; 22:129-140.

31. Malkin CJ, Pugh PJ, Jones RD, et al. The effect of testosterone replacement on endogenous inflammatory cytokines and lipid profiles in hypogonadal men J Clin Endocrinol Metab. 2004; 89:3313-8.

32. Chen J, Jiang Q, Xia X, et al. Individual variation of the SARS$\mathrm{CoV}-2$ receptor ACE2 gene expression and regulation. Aging Cell. 2020; 19:e13168.

33. Giagulli VA, Guastamacchia E, Magrone T, et al. Worse progression of COVID-19 in men: Is testosterone a key factor? Andrology. 2021; 9:53-64.

34. Çayan S, Uguz M, Saylam B, Akbay E. Effect of serum total testosterone and its relationship with other laboratory parameters on the prognosis of coronavirus disease 2019 (COVID-19) in SARSCoV-2 infected male patients: a cohort study. Aging Male. 2020; 23:1493-1503.

35. Rastrelli G, Di Stasi V, Inglese F, et al. Low testosterone levels predict clinical adverse outcomes in SARS-CoV-2 pneumonia patients. Andrology. 2021; 9:88-98.

36. Li W, Moore MJ, Vasilieva N, et al. Angiotensin-converting enzyme 2 is a functional receptor for the SARS coronavirus. Nature 2003; 426:450-454.

37. Hoffmann M, Kleine-Weber H, Schroeder S, et al. SARS-CoV-2 Cell Entry Depends on ACE2 and TMPRSS2 and Is Blocked by a Clinically Proven Protease Inhibitor. Cell. 2020; 181:271-280.e8.

38. Heurich A, Hofmann-Winkler H, Gierer S, et al. TMPRSS2 and ADAM17 cleave ACE2 differentially and only proteolysis by TMPRSS2 augments entry driven by the severe acute respiratory syndrome coronavirus spike protein. J Virol. 2014; 88:1293-1307.

39. Wambier CG, Goren A. Severe acute respiratory syndrome coronavirus 2 (SARS-CoV-2) infection is likely to be androgen mediated. J Am Acad Dermatol. 2020; 83:308-309.

40. Rozhivanov RV, Andreeva EN, Melnichenko GA, Mokrysheva NG. Androgens and Antiandrogens influence on COVID-19 disease in men. Probl Endokrinol (Mosk). 2020; 66:77-81.

\section{Correspondence}

Kadir Karkin, MD (Corresponding Author)

kadir_karkin@msn.com

Ergün Alma, MD

Health Sciences University, Adana City Training and Research Hospital,

Department of Urology, 01330, Adana (Turkey) 